\title{
Using multiple sensors to detect uncut crop edges for autonomous guidance systems of head-feeding combine harvesters
}

\section{$\operatorname{AUTHOR}(\mathrm{S}):$}

Cho, Wonjae; lida, Michihisa; Suguri, Masahiko; Masuda, Ryohei; Kurita, Hiroki

\section{CITATION:}

Cho, Wonjae ...[et al]. Using multiple sensors to detect uncut crop edges for autonomous guidance systems of head-feeding combine harvesters. Engineering in Agriculture, Environment and Food 2014, 7(3): 115-121

\section{ISSUE DATE:}

2014-07

URL:

http://hdl.handle.net/2433/189413

\section{RIGHT:}

(C) 2014 Asian Agricultural and Biological Engineering Association. Published by Elsevier B.V.; This is not the published version. Please cite only the published version.; この論文は 出版社版でありません。引用の際には出版社版をご確認ご利用ください。 


\title{
Using Multiple Sensors to Detect Uncut Crop Edges for Autonomous Guidance Systems of Head-Feeding Combine
}

\author{
Harvesters
}

\author{
Wonjae $\mathrm{CHO}^{* 1}$, Michihisa IIDA ${ }^{* 2}$, Masahiko SUGURI ${ }^{* 3}$, \\ Ryohei MASUDA $^{* 3}$, Hiroki KURITA ${ }^{* 3}$
}

\begin{abstract}
This study proposes a method for detection of uncut crop edges using multiple sensors to provide accurate data for the autonomous guidance systems of head-feeding combine harvesters widely used in the paddy fields of Japan for harvesting rice. The proposed method utilizes navigation sensors, such as a real-time kinematic global positioning system (RTK-GPS), GPS compass, and laser range finder (LRF), to generate a three-dimensional map of the terrain to be harvested at a processing speed of $35 \mathrm{~ms}$ and obtain the crop height. Furthermore, it can simultaneously detect the uncut crop edges by RANdom SAmple Consensus (RANSAC). The average of the lateral offset value and crop height of the uncut crop edge detected by the proposed method were $0.154 \mathrm{~m}$ and $0.537 \mathrm{~m}$, respectively.
\end{abstract}

[Keywords] Head-feeding combine harvester, Uncut crop edge detection, RTK-GPS, GPS compass, Laser range finder

\section{Introduction}

Crop harvesting in a field environment requires a high level of concentration because the operator has to simultaneously control the travel speed and direction of the harvesting machine while adjusting the height of the header after considering numerous parameters including crop height, biomass density, and other terrain conditions. However, it is challenging for the operator to maintain his or her health and physical condition because of the long hours operating the harvester under unfavorable environmental conditions such as the dust particles suspended in midair and noise and vibrations generated from the machine while harvesting. To overcome this challenge, autonomous guidance systems that perform the required tasks without human control are being developed to automatically steer the unmanned harvesters along the edges of uncut crops.

In contrast to a system that helps the operator control the harvesting during operation by guiding the harvesting machine along the prearranged target path, the autonomous guidance system performs unmanned harvesting and can be used to replace all field operations performed by the operator (Kise et al., 2005). The autonomous guidance system does not rely on the experience and proficiency of the operator and is able to
26 enhance work stability and productivity because the 27 system allows the harvesting machine to automatically 28 travel fast and accurately along the target path while 29 harvesting as it detects the surrounding environment. The 30 autonomous guidance system utilizes the following 31 functions for unmanned travel and harvesting. First, by 32 using the navigation sensors mounted on the harvesting 33 machine, the current position of the harvester is 34 estimated in real-time and uncut crop edges are detected 35 by sensing the surrounding environment. Next the target 36 path is determined together with the travel direction and 37 speed of the harvesting machine, which allows the 38 harvesting machine to travel and perform tasks without 39 damaging the crops. Finally, the harvest machine is 40 automatically steered, precisely running on the planned 41 target path. To fulfill these functions, the autonomous 42 guidance system requires on-board navigation sensors 43 and methodologies satisfying the sensor characteristics to 44 provide guidance information that can be used for path 45 planning and accurate steering.

46 In recent years, numerous sensor methodologies that 47 utilize various navigation sensors have been proposed 48 and developed for an autonomous guidance system of 49 harvesting machines, for example, global positioning 50 systems (GPSs), machine vision, laser range finders

*1 JSAM Student Member, Graduate School of Agriculture, Kyoto University, Kitashirakawa Oiwake-cho, Sakyo-ku, Kyoto, 606-8502, Japan; cho@elam.kais.kyoto-u.ac.jp

*2 JSAM Member, Corresponding author, Graduate School of Agriculture, Kyoto University, Kitashirakawa Oiwake-cho, Sakyo-ku, Kyoto, 606-8502, Japan; iida@elam.kais.kyoto-u.ac.jp

*3 JSAM Member, Graduate School of Agriculture, Kyoto University, Kitashirakawa Oiwake-cho, Sakyo-ku, Kyoto, 606-8502, Japan 
1 (LRFs), contact, and azimuth. The researchers at the National Agricultural Research Center (Japan) and Mitsubishi Farm Machinery Co., Ltd., developed an automatic traveling control system that can follow crop 5 rows and turn at the end of rows by detecting the uncut crops with a contact sensor and gyroscope mounted on 7 head-feeding combine harvesters (Sato et al., 1996). A team of researchers from the Carnegie-Mellon University and National Aeronautic and Space Administration 10 (NASA) used a color camera mounted on a hay 11 windrower to develop an automated guidance system 12 that can travel and perform harvesting tasks along the uncut crop edges detected in real-time, which was successfully tested for harvesting in a field of alfalfa (Ollis and Stentz, 1997). Scientists at Cemagref (France) proposed an automatic guidnace method that used laser sensors mounted on both windrow and combine harvesters (Chateau et al., 2000). Benson et al. (2003) used a monochrome camera mounted on a head directly above the uncut crop edge to develop and demonstrate a machine-vision-based guidance system for small-grain harvesters that can automatically steer by detecting uncut crop edges. Rovira-Más et al. (2007) proposed a method for detecting uncut crop edges that used stereoscopic vision for an autonomous guidance system for corn harvesters. The researchers at Kyoto University developed an autonomous guidance system that plans the target path and automatically steers using a real-time kinematic global positioning system (RTK-GPS) and GPS compass mounted on a head-feeding combine harvester along with the field information obtained prior to the harvest (Iida et al., 2012). The harvesting performance of the system was successfully tested in a paddy field.

These earlier researchers proposed sensor methodologies that can successfully detect uncut crop edges from the sensory data by using the inherent characteristics of sensors and applying them to the autonomous guidance systems. However, the sensor methodologies proposed by these researchers have limitations, depending on sensor characteristics. In the studies that utilized machine vision and LRFs mounted on harvesters for the detection of uncut crop edges (Ollis and Stentz, 1997; Chateau et al., 2000; Benson et al., 2003; Rovira-Más et al., 2007), the positions of the detected uncut crop edges were identified in relation to the mounted sensors. However, because the absolute position of the harvester could not be obtained from the mounted sensors, the locations of the detected uncut crop edges in the field were not identifiable. On the contrary,

51 in a study that configured the autonomous guidance 52 system by mounting on the harvester RTK-GPS detectors 53 that could acquire absolution positions (Iida et al., 2012), 54 the location of the harvester could be identified in the 55 field. However, because information about the upcoming 56 harvesting environment could not be acquired, the actual 57 harvesting was only limited to following pre-specified 58 paths.

59 To overcome the above constraints, this paper 60 proposes a method that uses multiple sensors to detect 61 uncut crop edges to provide data for autonomous 62 guidance systems of head-feeding combine harvesters 63 that are widely used in Japan for the harvesting of rice 64 and wheat crops. At a rapid processing speed of $35 \mathrm{~ms}$, 65 the proposed method uses navigation sensors, such as an 66 RTK-GPS, a GPS compass, and an LRF, mounted on a 67 head-feeding combine harvester to provide the current 68 position of the machinery and a three-dimensional (3D) 69 map of the terrain to be harvested, which are needed for 70 planning of the target path. Moreover, the uncut crop 71 areas can be separated from the ground areas, and the 72 edge and height of the uncut crops can be determined 73 from the generated 3D map data.

\section{Materials and Methods}

\section{Experimental setup}

\section{(1) Navigation sensors}

The navigation sensors used for this study were an RTK-GPS (Topcon Co., Ltd., Legacy-E+), a GPS compass (Hemisphere Co., Ltd., V110), an LRF (SICK AG, LMS111) and machine vision (Sensor Technology Co., Ltd., STC-TC33USB). As shown in Fig. 1, the navigation sensors (an RTK-GPS antenna and GPS compass) were mounted on the roof, the LRF was mounted on the upper front, and machine vision was installed in the ceiling inside the operation room of the head-feeding combine harvester (Mitsubishi Agricultural Machinery Co., Ltd., VY50CLAM). The sensors serve the following functions.

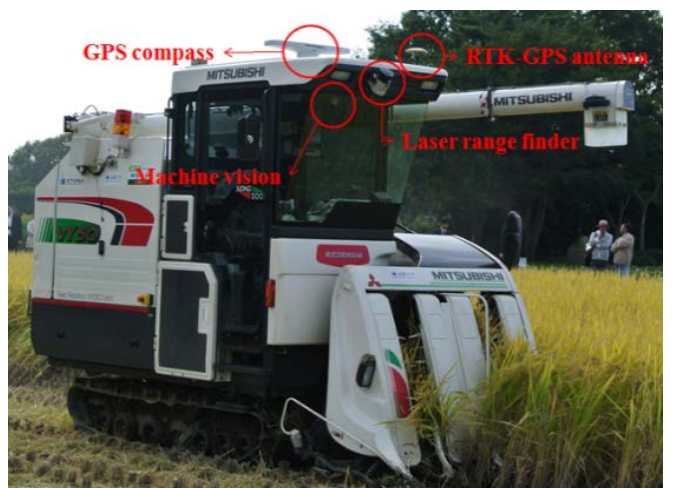


CHO, IIDA, SUGURI, MASUDA, KURITA :

Using Multiple Sensors to Detect Uncut Crop Edges for Autonomous Guidance Systems of Head-Feeding Combine Harvesters 3

Fig. 1 Navigation sensors mounted on the head-feeding combine harvester (modified from Iida et al., 2012).

An RTK-GPS was used to obtain the absolute and accurate position of the head-feeding combine harvester. 6 To compensate for the signal errors generated by the sensors, a virtual reference station (VRS) system was 8 used. A personal digital assistance device (HewlettPackard Co., Ltd., iPAQ hx2190b) and mobile

\begin{tabular}{cc}
\hline Model & LMS111 \\
\hline Maximum field of view $\left(^{\circ}\right)$ & 270 \\
Angular resolution $\left(^{\circ}\right)$ & $0.25,0.50$ \\
Maximum scanning range $(\mathrm{m})$ & 20 \\
Rotation frequency $(\mathrm{Hz})$ & 25,50 \\
Data interface type & Ethernet, RS-232, CAN
\end{tabular}

Although machine vision was not used for the detection of uncut crop edges, it was used for visual recording and storing of the traveling and harvesting tasks of the head-feeding combine harvester. The machine vision used in this study used CCD image sensors and supports a USB 2.0 interface. The sensor also acquired color images with a 640 pixels (horizontal) by 480 pixels (vertical) resolution, at a speed of 10 FPS. The lens (Pentax Ricoh Imaging Co., Ltd., FL-CC815B-VG) installed on the machine vision system
45 had a field of view of $56.5^{\circ}$. For this study, the acquired 46 color images were saved in the audio video interactive 47 format.
48

\section{(2) Control system}

Two electronic control units (ECU), named ECU-KU1 and ECU-KU2, were developed to integrate the control of the components of the head-feeding combine harvester and the navigation sensors mounted on the harvester, and the pre-existing control system for the head-feeding combine harvester was remodeled (Iida et al., 2012). As shown in Fig. 2, ECU-KU1 communicates with the components of the head-feeding combine harvester, and ECU-KU2 with the navigation sensors (RTK-GPS and GPS compass). ECU-KU1 and ECU-KU2 communicate with one another via the control area network bus. The personal computer (PC) used for integrated system control communicates with ECU-KU1 via the RS-232C ports, while the LRF communicates with the PC via Ethernet, and the machine vision via USB port.

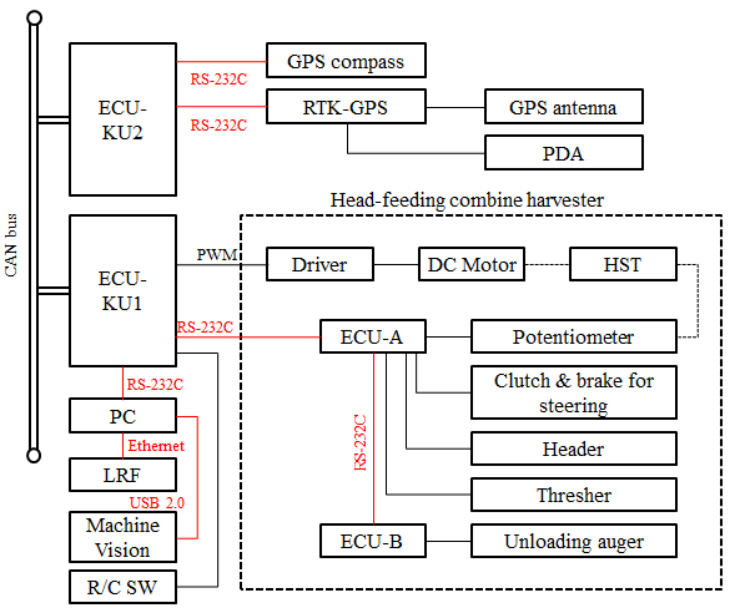

Fig. 2 Control system architecture of head-feeding combine harvester.

\section{(3) Integrated sensor control platform}

The study implemented a guidance method using multiple sensors by introducing the integrated sensor control platform (ISCP), currently developed by our project team for head-feeding combine harvesters. ISCP supports various navigation sensors, such as machine vision, LRFs, and GPSs that are mainly used for the autonomous guidance systems of head-feeding combine harvesters, and they can also display real-time sensor data with a graphical user interface. In addition, the open-source platform can be freely modified and re-distributed without license restrictions (https://github.com/FiroKyoto/IntegratedSensorControlPlatform.git). 
1 In this study, ISCP was used to obtain data about the components and navigation sensors of the head-feeing 3 combine harvester from ECU-KU1, ECU-KU2, the machine vision, and LRF at a frequency of $10 \mathrm{~Hz}$.

\section{3D terrain mapping}

Because rice is planted evenly in $0.3 \mathrm{~m}$ inter-row $\left(d_{r}\right)$ and $0.2 \mathrm{~m}$ intra-row $\left(d_{c}\right)$ intervals in the paddy fields of Japan, as shown in Fig. 3, each row is parallel to its neighbors. Because the head-feeding combine harvester performs its harvesting task while traveling along the uncut crop edge, the precise detection of the crop edge is pivotal to the performance enhancement of the autonomous guidance system. The $2 \mathrm{D}$ profile data from the LRF, the heading angle data from the GPS compass, and the absolute position data from the RTK-GPS, all obtained from the sensors mounted on the head-feeding combine harvester, were used to generate a 3D map of the to be harvested terrain and to obtain location of the uncut crop edge and crop height.

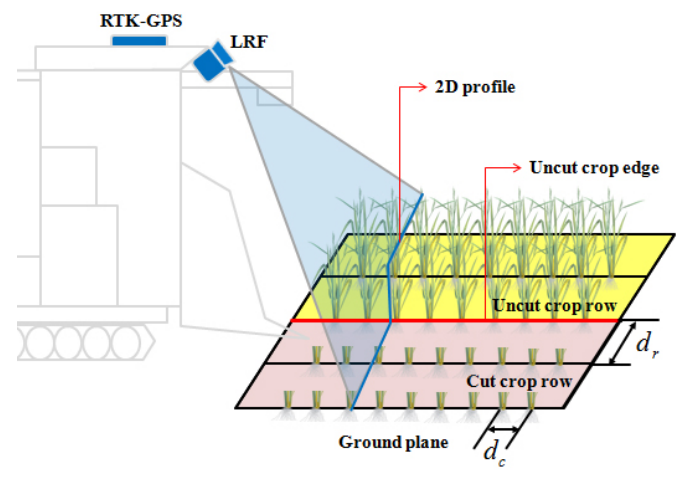

Fig. 3 Rows of rice plants in a paddy field.

Fig. 4 depicts the extrinsic parameters of the navigation sensors mounted on the head-feeding combine harvester. Parameters $\theta_{L R F}$ and $h_{L R F}$ are defined as the tilt angle and height of the installed LRF from the ground, which are $50.5^{\circ}$ and $2.5 \mathrm{~m}$, respectively. The longitudinal distance $\left(l_{a}\right)$ and transverse distance $\left(l_{c}\right)$ of the LRF from the origin of the $x y$ plane of the 3D Euclidean space $(W=\{(x, y, z)\} \in$ $\left.E^{3}\right)$, configured with the center of the RTK-GPS as the base point, are $0.45 \mathrm{~m}$ and $0.59 \mathrm{~m}$, respectively. Moreover, the longitudinal distance $\left(l_{b}\right)$ and transverse distance $\left(l_{d}\right)$ of the divider of the right-side end of the header from the center of the LRF are $0.6 \mathrm{~m}$ and $0.07 \mathrm{~m}$, respectively. The GPS compass is installed in parallel to the $x y$ plane of $W$ space and calculates the angle between the GPS compass in the clockwise direction, which is aligned to the magnetic north direction of the

41 Earth, and the $y^{\prime}$ axis. Therefore, the heading angle $(\varphi)$

42 of the head-feeding combine harvester can be obtained

43 by adding $+90^{\circ}$ to the angle obtained above. The center

44 of the lens installed as part of the machine vision is

45 located $2.3 \mathrm{~m}$ perpendicular to the ground surface $\left(h_{M V}\right)$

46 at a tilt angle $\left(\theta_{M V}\right)$ of $38.5^{\circ}$. Moreover, because the 47 machine vision is positioned on the $y z$ plane, together 48 with the LRF, both centers of the machine vision lens 49 and LRF are positioned on the $y z$ plane.

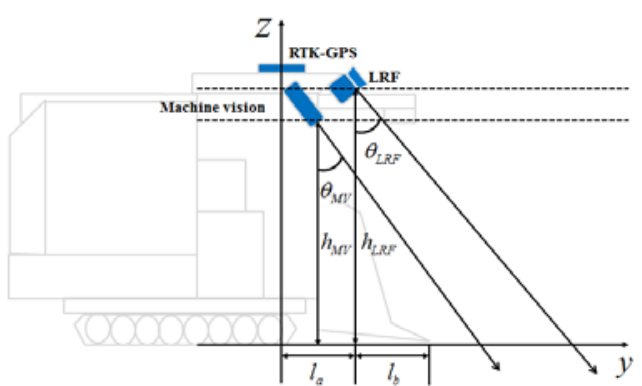

(a) $y z$ plane.

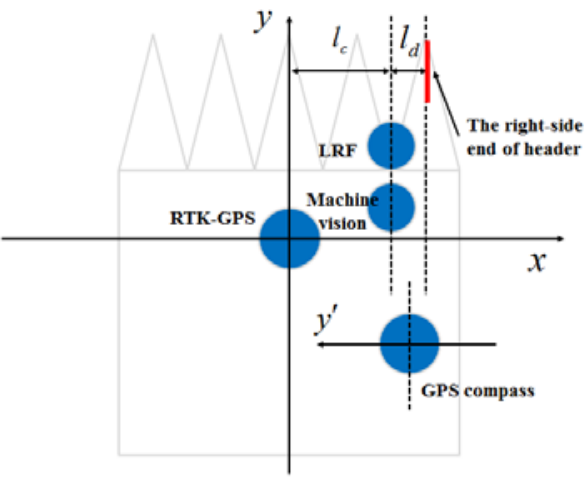

(b) $x y$ plane.

Fig. 4 Extrinsic parameters of the navigation sensors.

During the process of transformation and integration, a $3 \mathrm{D}$ terrain map is generated from the data acquired by the navigation sensors mounted on the head-feeding combine harvester, and it has the mathematical relationship, as shown in Fig. 5.

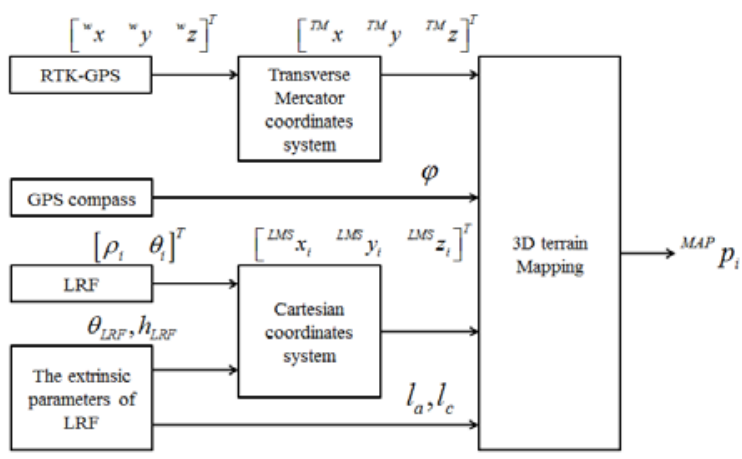

Fig. 5 Mathematical relationship between sensors. 
CHO, IIDA, SUGURI, MASUDA, KURITA :

Using Multiple Sensors to Detect Uncut Crop Edges for Autonomous Guidance Systems of Head-Feeding Combine Harvesters 5

First, the data in the polar coordinates system acquired from the LRF and extrinsic parameters $\left(\theta_{L R F}, h_{L R F}\right)$ of the LRF are substituted in Eq. (1), and then converted into data in the Cartesian coordinates system, which can be expressed in $W$ space. Next, the absolute position 6 data acquired from the RTK-GPS is converted to data in the transverse mercator coordinates system, which assumes the absolute position of the RTK-GPS mounted on the harvester at the beginning of the harvesting task as

the center of origin. Lastly, the sensory data converted by the above process, heading angle $(\varphi)$ of the harvester, and extrinsic parameters $\left(l_{a}, l_{c}\right)$ of the LRF are substituted in Eq. (2) and then converted into data in the Cartesian coordinates system, which can be expressed in $W$ space. The converted data is consecutively saved in a dynamic array until the head-feeding combine harvester reaches the end of the row.

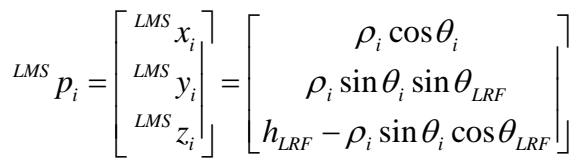

where

$\theta_{i}$ is the measurement angle acquired from the LRF, $\rho_{i}$ is the reflection distance acquired from the LRF, and $\theta_{L R F}$ and $h_{L R F}$ are the tilt angle and the height of the LRF from the ground, respectively.

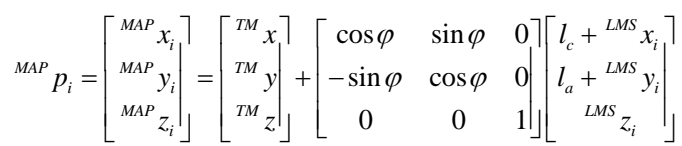

where

${ }^{T M} x,{ }^{T M} y,{ }^{T M} Z$ are values acquired from the RTK-GPS converted into values in the transverse mercator coordinates system, $\varphi$ is the heading angle of the head-feeding combine harvester, and $l_{a}$ and $l_{c}$ are the longitudinal and transverse distances from the RTK-GPS to the LRF.

The LRF data transformed into Cartesian coordinate
40 the perpendicular to the data set and the segment becomes maximum, by using Eq. (3) (Kimberling, 1998). Because the resulting $p_{k}$ denotes the outermost boundary point of the uncut crop area, as shown in Fig. 6, the uncut crop area and ground area can be discriminated based on $p_{k}$.

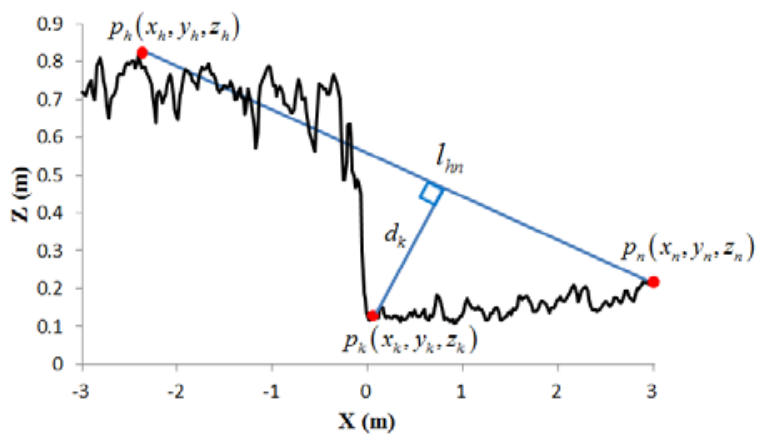

Fig. 6 LRF data distribution expressed on $x z$ plane.

$$
d_{k}=\frac{\left|\left(x_{n}-x_{h}\right)\left(z_{h}-z_{k}\right)-\left(x_{h}-x_{k}\right)\left(z_{n}-z_{h}\right)\right|}{\sqrt{\left(x_{n}-x_{h}\right)^{2}+\left(z_{n}-z_{h}\right)^{2}}}
$$

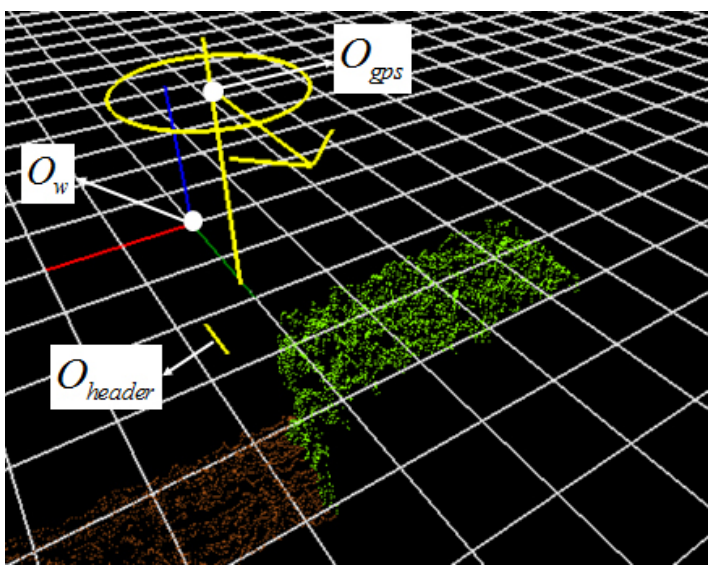

Fig. 7 3D terrain map using OpenGL.

Fig. 7 shows the 3D terrain map obtained when applying the proposed method. The 3D terrain map is expressed by using Open Graphics Library (OpenGL) application programming interface (API). OpenGL is an API that performs the rendering of 3D objects, such as lines, rectangles, and other primitive graphic elements, and projects the results onto a 2D screen (Shreiner, 2009). Every time ${ }^{M A P} P_{i}$ data is added onto the dynamic array, which is used for storing ${ }^{M A P} P_{i}$, the glVertex3f function, which is provided for the conversion of the data into $3 \mathrm{D}$ point expression in OpenGL, is used to express in real-time the data value of ${ }^{M A P} P_{i}$ in the 3D point format in space $W$ of OpenGL. $O_{w}$ signifies the coordinate system of space $W$, which is configured using the 
1 absolute position obtained from the RTK-GPS at the start of harvesting as the origin. $O_{g p s}$ and $O_{\text {header }}$ refer to 3 the center of the RTK-GPS and right-side end of the header, respectively. Additionally, the 3D points of the 5 uncut crops and ground are expressed in green and brown, respectively. performs samplings of the minimum data required for determining the model parameters from the total original data and calculates the values repeatedly to find the optimal values.

The original data applied to the RANSAC algorithm is the previously calculated set of the outermost boundary points $\left(p_{k}\right)$ of the uncut crop area, configured in a dynamic array. The dynamic array is initialized at the start of harvest and consecutively adds $p_{k}$ until the head-feeding harvester reaches the end of the row. In this case, $z$ values from $p_{k}$ are not used because the uncut crop edge exists on the $x y$ plane of space $W$. Furthermore, $p_{k}$ is determined as an inlier where the perpendicular distance value of $p_{k}$ to the direct line of the model is smaller than the threshold value and an outlier if bigger. In this study, the threshold value was set as 0.1 . The number of repeats $(\bar{N})$ of the RANSAC algorithm can be calculated from Eq. (4). In this study, $\bar{N}$ was determined by setting the number of sample data $(\bar{m})$ as 5 , the probability $(\bar{p})$, where at least one sample set included valid data, such as 0.99 , and the probability $(\bar{\mu})$ of the data validity as 0.5 . The RANSAC algorithm is then given as follows:

8 until Maximum $\bar{N}$ iterations reached

9 return Best-fit straight line model

39

$$
\bar{N}=\frac{\log (1-\bar{p})}{\log \left(1-\bar{\mu}^{\bar{m}}\right)}
$$

40

\footnotetext{
RANSAC algorithm

1 Initial: A set of $N$ points in dynamic array

2 repeat

3 Choose a sample of $m$ points uniformly at random

$4 \quad$ Fit a straight line model through the $m$ points

5 Compute the perpendicular distance of other points to the straight line model

$6 \quad$ Construct the inlier set

7 If there are enough inliers, re-compute the straight line model parameters, store the straight line, and remove the inliers from the set
}

Fig. 8 shows the results of determining the uncut crop edge from the 3D terrain map by using the RANSAC algorithm. The uncut crop edge was determined and updated by the RANSAC algorithm every time additional data was received from the navigation sensors until the head-feeding combine harvester reached the end of the row. As can be observed in Fig. 8, the uncut crop edge is expressed as a red line on the $3 \mathrm{D}$ terrain map, which can be accurately determined by RANSAC.

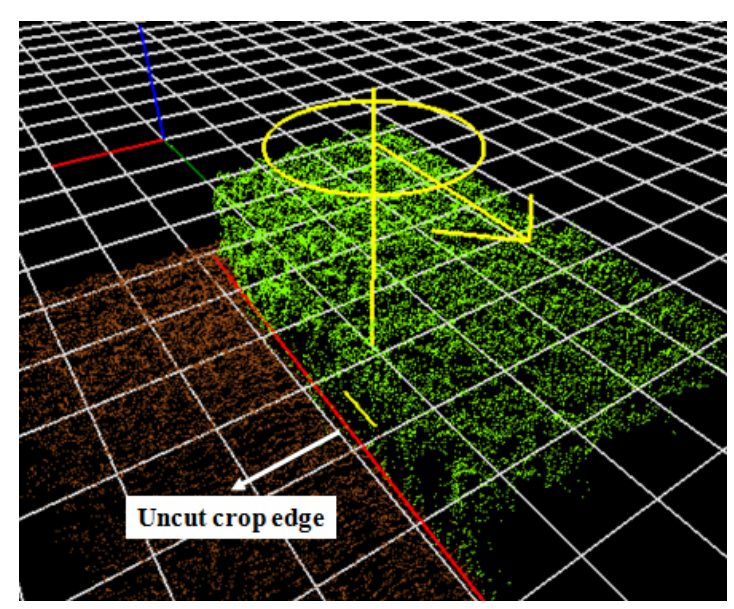

Fig. 8 Uncut crop edge detection by RANSAC.

\section{Experimental method}

To evaluate the performance of the proposed method, data from the navigation sensors were obtained during the harvest operations of a head-feeding combine harvester controlled by an operator in a rice paddy field in Nantan City, Kyoto Prefecture, Japan. The head-feeding combine harvester harvested rice along target paths at an average travel speed of $0.6 \mathrm{~m} / \mathrm{s}$. Because the head-feeding combine harvester performed its operations while traveling counter clockwise, as shown in Fig. 9, the sensor data obtained were categorized into four datasets according to the movement directions.

In this study, the proposed method was used to evaluate the performance of the determination of the uncut crop edge from each section. First, target paths that functioned as the baseline for each determination zone were set. Then, the lateral offset was calculated between the determined uncut crop edges and baseline target paths. As shown in Fig. 9, the target path for each zone is 
CHO, IIDA, SUGURI, MASUDA, KURITA :

Using Multiple Sensors to Detect Uncut Crop Edges for Autonomous Guidance Systems of Head-Feeding Combine Harvesters 7

1 a single straight line, which is derived by connecting the

$23 \mathrm{D}$ points on the right-side end of the headers expressed

3 on the 3D terrain map at the beginning and end-of-row time points of the harvesting task. Because the straight

5 line is expressed on the $x y$ plane, $z$ values are not used from the 3D points obtained above. To derive the target 7 path from each harvest zone, an experienced operator 8 accurately operated the combine in a paddy field filled with rice plants planted at regular intervals. The combine 10 traveled at a speed of $0.6 \mathrm{~m} / \mathrm{s}$, lower than the normal 11 harvest travel speed of $1.0 \mathrm{~m} / \mathrm{s}$, and followed the uncut

crop row to perform the harvesting task.

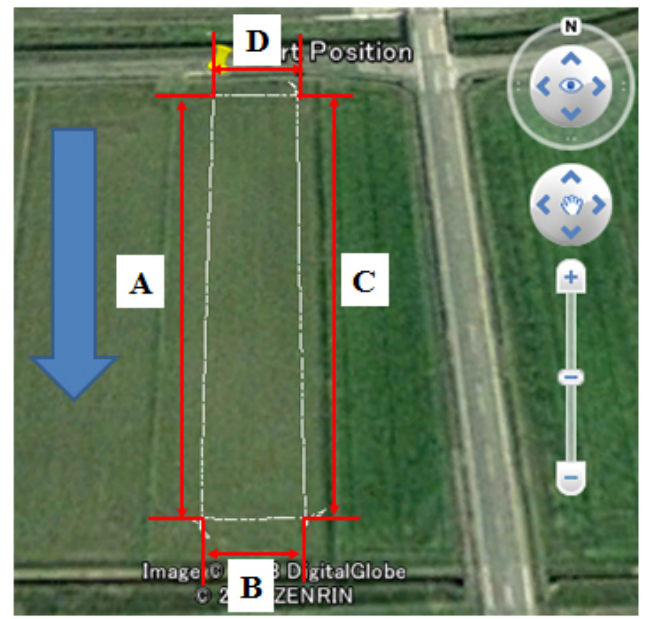

Fig. 9 Traveling path of the head-feeding combine harvester (image from Google Maps).

\section{Results and Discussion}

Fig. 10 shows the result of a 3D terrain map generated with the navigation sensor data obtained using the proposed method. The movie clip of the results can be accessed via the following address (http://youtu.be/juyYuQfvgkk). The proposed method, at an average processing speed of $35 \mathrm{~ms}$, acquires data from the mounted sensors, generates a 3D terrain map by using the sensory data, and simultaneously determines the uncut crop edge and average crop height from the 3D terrain map.

The average crop height obtained for each harvest section under the proposed method and lateral offset values of the target path to the determined uncut crop edge are summarized in Table 2. Fig. 11 shows the target paths set for each section as well as the result of the uncut crop edge determined by RANSAC. The target path and the uncut crop edge are expressed as blue and red lines, respectively, while the set of boundary points applied by RANSAC are shown in gray. In Fig. 11, the $x$-axis refers to the distance traveled by the head-feeding combine harvester while performing the harvesting task, starting from the absolute position acquired from the RTK-GPS mounted on the head-feeding combine harvester at the start of the harvest as the center of origin. The $y$-axis signifies the perpendicular distance from the determined values (the set of boundary points and uncut crop edge) to the assumed target path, obtained by using the proposed method. Regarding the signs of $y$-axis values, they will be negative (-) when the determined values are located on the left side of the target path in the traveling direction of the head-feeding combine harvester on the 3D terrain map, and positive $(+)$ when located on the right side of the target path. The lateral offset for each zone, shown in Table 2, indicates the average of $y$-axis values of the uncut crop edge, as shown in Fig. 11 . Moreover, the "average crop height" for each zone refers to the average of the $z$ values of the 3D points (expressed in green in Fig. 10) that are distinguished as uncut crop area of the generated 3D terrain map.

Table 3 shows the average perpendicular distances of the determined uncut crop edge from the set of boundary points used for RANSAC and the inlier ratio of the set of boundary points for each harvest section. The inlier ratio, shown in Table 3, refers to the ratio of the number of elements out of the set of boundary points stored in the dynamic array that have perpendicular distances to the uncut crop edges within the inlier range. When the perpendicular distance is smaller than the threshold value, the boundary point is regarded as an inlier, and regarded as an outlier if bigger. In this study, the threshold value is set at 0.1 for the proposed uncut crop edge detection method. As the value for the inlier ratio increase, the number of elements out of the set of boundary points, determined by RANSAC with the uncut crop edge as the basis, is dispersed in high densities. As shown in Fig. 11, the uncut crop edge determined for each section is located on the right side of the target path, as viewed from the perspective of the traveling direction of the head-feeding combine harvester, and the average lateral offset to the target path is $0.154 \mathrm{~m}$. Table 2 Results using the proposed method.

\begin{tabular}{cccc}
\hline Dataset & $\begin{array}{c}\text { Movement } \\
\text { Direction }\end{array}$ & $\begin{array}{c}\text { Average } \\
\text { Crop Height [m] }\end{array}$ & $\begin{array}{c}\text { Lateral } \\
\text { Offset [m] }\end{array}$ \\
\hline A & South & 0.514 & 0.294 \\
B & East & 0.503 & 0.139 \\
C & North & 0.549 & 0.067 \\
D & West & 0.580 & 0.119 \\
\hline Average & & 0.537 & 0.154
\end{tabular}


1

2

\begin{tabular}{ccc}
\hline Dataset & $\begin{array}{c}\text { Average } \\
\text { Perpendicular Distance [m] }\end{array}$ & $\begin{array}{c}\text { Inlier } \\
\text { Ratio [\%] }\end{array}$ \\
\hline A & 0.070 & 74.4 \\
B & 0.052 & 90.2 \\
C & 0.072 & 78.2 \\
D & 0.059 & 83.6 \\
\hline Average & 0.063 & 81.6
\end{tabular}

3

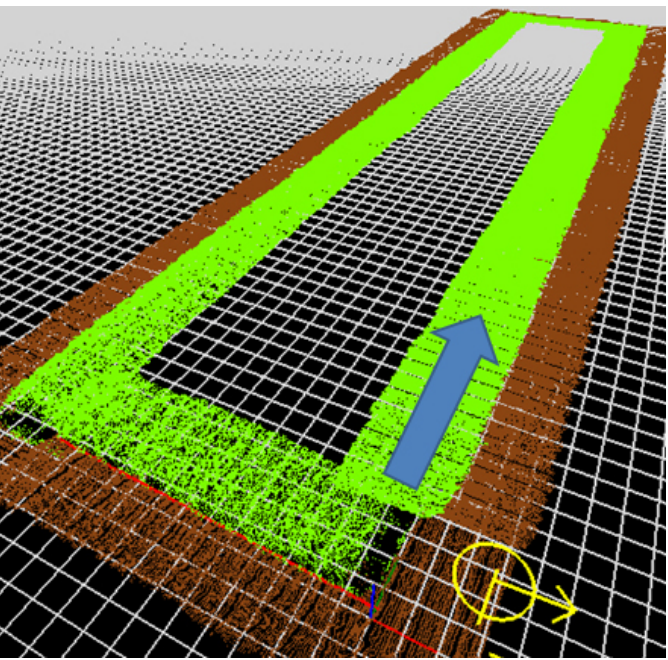

Fig. 10 3D terrain map of the experiment field.

6

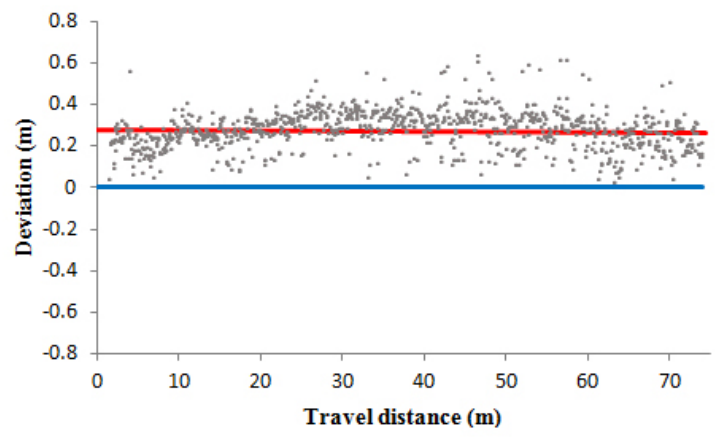

(a) Section A.

9

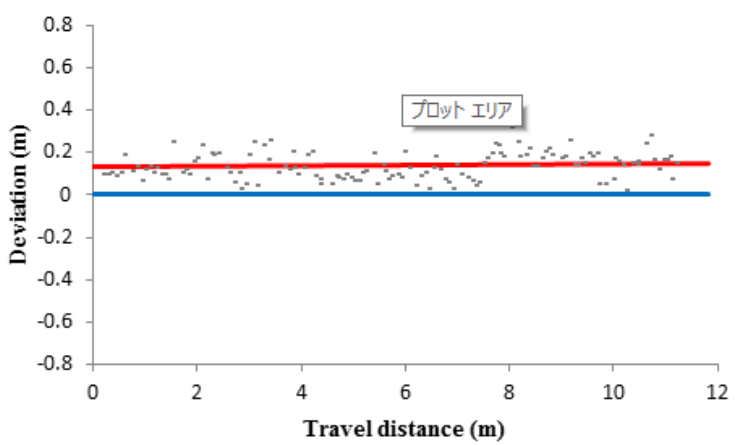

(b) Section B.
13

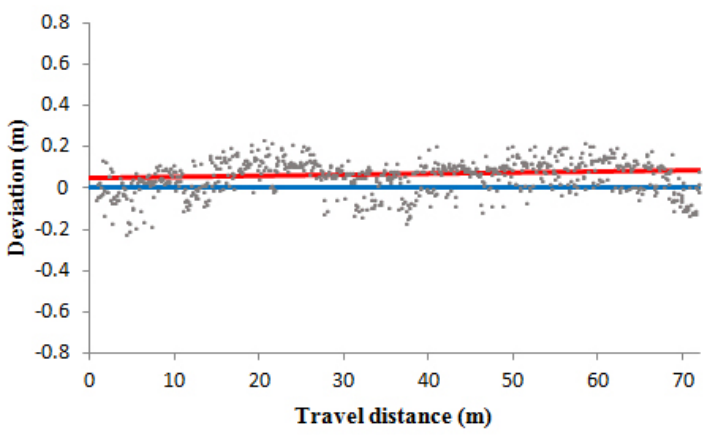

(c) Section C.

16

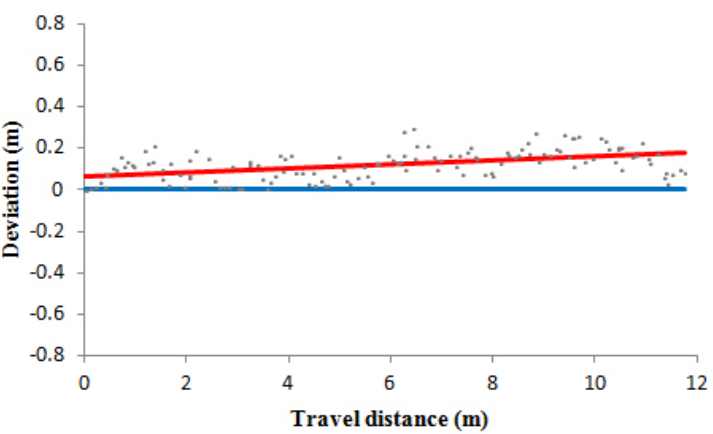

(d) Section D.

Fig. 11 Target path and the uncut crop edge determined

by RANSAC.

The main reason for the lateral offset between the determined uncut crop edge and target path when applying the proposed method can be explained from Fig. 12. The paddy field image in Fig. 12 was acquired from the machine vision, which was mounted on the head-feeding combine harvester that was performing the harvest task in Section A. When performing the task in Section A, the harvester was operated accurately, following the target path (blue line), as perceived by an experienced operator. Therefore, the right-side end of the header can be considered to be located on the straight 33 line of the target path, as shown in Fig. 12. Moreover, the 34 red line shown in Fig. 12 signifies the row of crops 35 already harvested that is located on the right-side of the 36 target path. Because an inter-row interval of rice plants in 37 a Japanese paddy field is $0.3 \mathrm{~m}$, the lateral offset of the 38 red line to the target path is $0.3 \mathrm{~m}$. The lateral offset 39 value between the determined uncut crop edge and target 40 path in Section A is $0.294 \mathrm{~m}$, which is almost identical to 41 the lateral offset value $(0.3 \mathrm{~m})$ between the red line and 42 the target path shown in Fig. 12. As described, the lateral 43 offset is caused by the lodging of the uncut crops, which 44 obscures the region between the target path and red line, 
CHO, IIDA, SUGURI, MASUDA, KURITA :

Using Multiple Sensors to Detect Uncut Crop Edges for Autonomous Guidance Systems of Head-Feeding Combine Harvesters 9

1 thereby making it difficult for the LRF to scan the region from the location where it is mounted $\left({ }^{L R F} O_{a}\right)$. To resolve the above issue, the mounting location of the 4 LRF needs to be moved to the right-side $\left({ }^{L R F} O_{b}\right)$ on the 5 right-side end of the header.

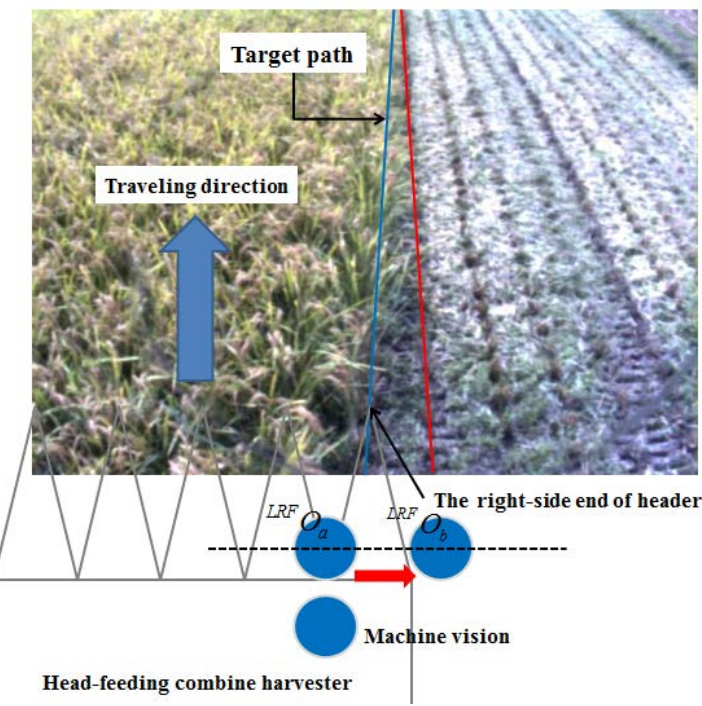

Fig. 12 Paddy field image acquired during harvesting task from Section A and relative position of the head-feeding combine harvester.

\section{Summary and Conclusions}

This study proposed a method to detect uncut crop edges using multiple sensors to provide accurate data for the autonomous guidance systems of head-feeding combine harvesters that are widely used in the paddy 6 fields of Japan for harvesting rice. The proposed method 17 was able to generate $3 \mathrm{D}$ maps of the terrain to be the uncut crop edge in general, its performance displayed a tendency to decline when the target path was obscured by the lodging of rice plants. Therefore, to enhance the performance of the proposed method, the mounting position of the LRF needs to be modified to allow for a more accurate scanning of the target path, together with the addition of an algorithm that can adjust for the error in results.
Benson, E. R., J. F. Reid and Q. Zhang. 2003. Machine vision-based guidance system for agricultural grain harvesters using cut-edge detection. Biosystems Engineering 86(4): 389-398.

Chateau, T., C. Debain, F. Collange, L. Trassoudaine and J. Alizon. 2000. Automatic guidance of agricultural vehicles using a laser sensor. Computers and Electronics in Agriculture 28(3): 243-257.

Fischler, M. A. and R. C. Bolles. 1981. Random sample consensus: a paradigm for model fitting with applications to image analysis and automated cartography. Communications of the ACM 24(6): 381-395.

Iida, M., R. Uchida, H. Zhu, M. Suguri, H. Kurita and R. Masuda. 2012. Path-following control for a head-feeding combine robot. Engineering in Agriculture, Environment and Food 6(2): 61-67.

Kimberling, C. 1998. Triangle centers and central triangles: Utilitas Mathematica Publishing, Inc.

Kise, M., Q. Zhang and F. Rovira Más. 2005. A stereovision-based crop row detection method for tractor-automated guidance. Biosystems Engineering 90(4): 357-367.

Ollis, M. and A. Stentz. 1997. Vision-based perception for an automated harvester. In Proc. IEEE/RSJ International Conference on Intelligent Robots and Systems, 1838-1844. September.

Rovira-Más, F., S. Han, J. Wei and J. F. Reid. 2007. Autonomous guidance of a corn harvester using stereo vision. Agricultural Engineering International: the CIGR Ejournal 9.

Sato, J., K. Shigeta and Y. Nagasaka. 1996. Automatic operation of a combined harvester in a rice field. In Proc. IEEE/SICE/RSJ International Conference on Multisensor Fusion and Integration for Intelligent Systems, 86-92. Washington, DC, 8-11 December.

Shreiner, D. 2009. OpenGL programming guide: The official guide to learning OpenGL, Versions 3.0 and 3.1: Pearson Education.

(Received : X. January. 20XX, Accepted : X. February. 20XX) 